\title{
Laos struggles to lower maternal mortality
}

$\mathrm{W}$ hen 32-year-old Bouavanh Songmala was growing up in a remote village in Laos, death during child birth was all too common. United Nations (UN) agencies estimated there were 1215 such deaths per 100000 live births in 1990. At the time, Laos had few midwives; none had been trained between 1987 and 2010, mainly due to lack of funds.

Bouavanh recently qualified as a male midwife in this southeast Asian nation, joining the ranks of 800 midwives and skilled birth attendants. Despite this improvement, more than half of women still lack supervised births, and Laos' maternal mortality rate remains among the worst outside of Africa. UN agencies and the Ministry of
Health estimate there are still between 220 and 370 deaths per 100000 live births. Laos lags behind other southeast Asian countries, such as Vietnam, which has a maternal mortality rate of 49 deaths per 100000 births. Canada's rate is 11 per 100000 .

Undeniably, Laos has made progress. The World Health Organization said in May that Laos was 1 of 11 countries that has achieved a $75 \%$ reduction in maternal mortality since 2000. But there is uncertainty over the accuracy of statistics, because birth and death registers are not kept, and, according to a May 2014 article in The Lancet, determining what constitutes a maternal death is not straightforward.

Laos is aiming to achieve the UN's
Millennium Development Goal 5: a $5.5 \%$ annual rate of reduction in maternal deaths and a three-quarters drop on 1990 levels. But UN agencies say Laos' target of 260 deaths per 100000 live births by 2015 is "unlikely," a conclusion shared by a study published in December.

Part of the reason for this pessimism is unreliable numbers and a myriad factors affecting progress. The increase in midwives and skilled birth attendants has certainly helped; skilled attendants are now present at $41.5 \%$ of births, and more than half of pregnant Laotian women receive skilled antenatal care at least once, says the UN Population Fund (UNFPA), which is helping to train these workers. It expects the num-

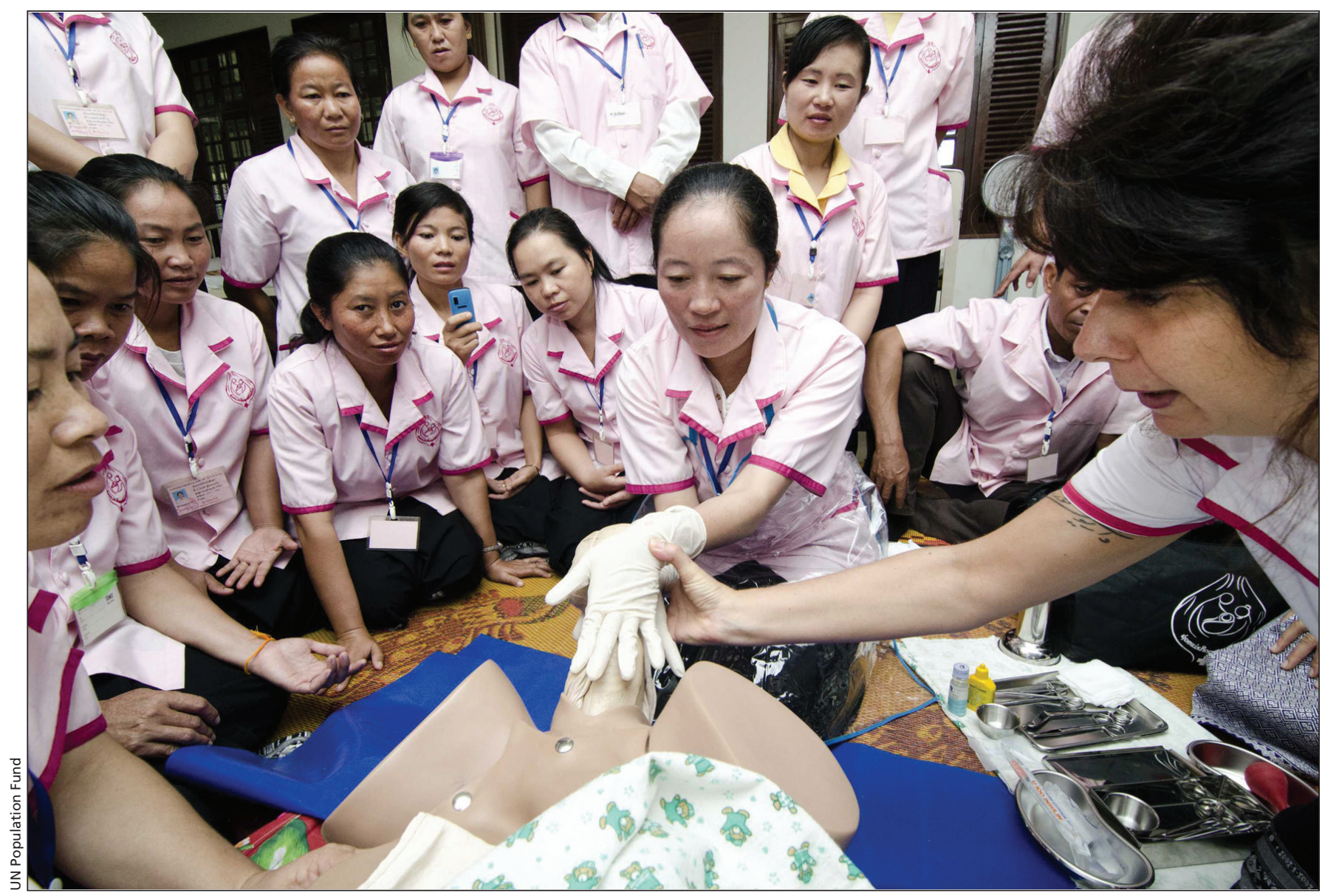

The number of midwives and skilled birth attendants in Laos is expected to nearly double, to 1500 , by 2015 , owing to training support from the UN Population Fund. 
ber of personnel to nearly double, to 1500, next year. Continued investment and support for midwives will be crucial in helping Laos escape decades of high maternal death rates, says Siriphone Sally Sakulku, UNFPA's reproductive health program coordinator in the Laos capital of Vientiane. "Laos needs midwives more than ever."

Birth spacing and improved socioeconomics also seem to play a role in improved maternal mortality. Fertility has fallen from six to three births per woman since 1990; however, health workers in Vientiane say access to contraception hasn't improved substantially.

UN agencies suggest Laos' economic growth rate of around $8 \%$ in recent years - among the highest in the world and attendant rising incomes have also helped to reduce maternal deaths.

On the other hand, only $3.7 \%$ of deliveries are by cesarean, and abortion remains illegal.

In addition, accessing people in remote areas is hugely problematic, particularly during the monsoon, when roads are frequently washed out.

Australia's Burnet Institute, which works in eastern Laos, says medical facilities are lacking, and medical professionals often refuse to work in remote areas. Christi Lane-Barlow, Burnet's country representative, says, "The level of disparity in terms of what people can access is really, really severe." - Steve Finch, Vientiane, Laos

CMAJ 2014. DOI:10.1503/cmaj.109-4833 